\title{
THE INDICATION OF RESECTION-RECONSTRUCTION WITH TUMOR PROSTHESIS FOR THE YOUNG PATIENT WITH OSTEOSARCOMA
}

doi: $10.2478 /$ rojost-2018-0028

\author{
A. Cursaru12, A.G. Lupu'12, D. Anghelescu1, G. Mardare ${ }^{1}$, R. Ene ${ }^{12}$, C. Cirstoiu'12 \\ ${ }^{1}$ University Emergency Hospital, Bucharest, Romania \\ 2"Carol Davila" University of Medicine and Pharmacy, Bucharest, Romania
}

Introduction. The osteosarcoma represents the most frequently encountered primitive malignant bone tumor, representing 30\% of the malignant bone tumors with an unpredictable evolution.

Materials and methods. A retrospective study realized over a period of 3 years (Jan. 2016-Jan. 2018), which included 6 patients diagnosed with osteosarcoma and treated through the reconstruction with tumor prosthesis technique in the Orthopaedics and Traumatology Clinic of the University Emergency Hospital in Bucharest.

Results. Of the total 6 patients, 5 were male and 1 was a female with ages between 20 and 61 years old, with an average of 30.6 years. The localization of the tumor was at the distal femur ( 3 cases) and at the proximal tibia (3 cases).

In 3 cases, the reattachment of the extensor apparatus of the knee to the prosthesis was needed, while for 3 of the cases, a musculocutaneous flap was created for the coverage of the implant.

All the 6 patients were monitored and had postoperative follow-ups at 3,6 and 12 months by having clinical and imagistic evaluations in search of the eventual local recurrences or metastases in other tissues and organs. The postoperative evaluation of the function of the joint was realized with the Knee Society Score Questionnaire and recorded very good results (76-91) with an average of 82 points.

Conclusions. The short-term results of this study about the treatment of the knee osteosarcoma with modular prostheses show that this treatment has a low tumoral recurrence rate and restores the joint function.

Keywords: tumor prosthesis, osteosarcoma, resection-reconstruction 